\title{
Exploring Factors that Influence Individuals' Choice Between Internal Combustion Engine Cars and Electric Vehicles
}

\author{
Dominik Bucher ${ }^{1}$, Henry Martin ${ }^{1}$, Jannik Hamper ${ }^{1}$, Atefeh Jaleh ${ }^{2}$, \\ Henrik Becker ${ }^{2}$, Pengxiang Zhao ${ }^{1}$ and Martin Raubal ${ }^{1}$ \\ ${ }^{1}$ Institute of Cartography and Geoinformation, ETH Zurich \\ ${ }^{2}$ Institute for Transport Planning and Systems, ETH Zurich \\ \{dobucher, martinhe, hamperj, pezhao, mraubal\} @ethz.ch \\ henrik.becker@ivt.baug.ethz.ch, atefe.jaleh@gmail.com
}

\begin{abstract}
The adoption of electric vehicles has the potential to help decarbonizing the transport sector if they are powered by renewable energy sources. Limitations commonly associated with e-cars are their comparatively short ranges and long recharging cycles, leading to anxiety when having to travel long distances. Other factors such as temperature, destination or weekday may influence people in choosing an e-car for a certain trip. Using a unique dataset of 129 people who own both an electric vehicle (EV) as well as one powered by an internal combustion engine (ICE), we analyze tracking data over a year in order to have an empirically verified choice model. Based on a wide range of predictors, this model tells us for an individual journey if the person would rather choose the EV or the ICE car. Our findings show that there are only weak relations between the predictor and target variables, indicating that for many people the switch to an e-car would not affect their lifestyle and the related range anxiety diminishes when actually owning an electric vehicle. In addition, we find that choice behavior does not generalize well over different users.
\end{abstract}

Keywords: Electric Vehicle, Transport Mode Choice, Mobility.

\section{Introduction}

Individual fossil fuel based transportation is a major contributor to greenhouse gas (GHG) emissions and thereby a serious threat to the global ecosystem $[8,36,16]$. With the imminent threat of climate change, the decarbonization of individual transportation has become one of the important questions of our time. In that context, renewable energy generation combined with electric vehicles (EVs) has great potential as it leads to significantly lower GHG emissions than internal combustion engine (ICE) cars [33, 14, 12]. In line with this, many major car manufacturers started adding EVs to their product range and the share of EVs in global car sales is projected to be at around $35 \%$ by 2040 [19]. However, while electrification is currently the most promising path towards a more sustainable transportation sector, the large-scale adaption of EVs is a slow process and people still associate EVs with uncertainties: "How far can I drive with a fully charged vehicle? Will I have charging stations on the way to my destination? How 
quickly does the battery wear out?" Such questions are commonly at the center of discussion when someone considers switching to an EV. As a result of this mistrust, EVs are often bought as an additional second car, complementary to the ICE car [13]. The resulting group of people is over-equipped with mobility tools and potentially has to decide for every trip whether to use the EV or the ICE car. A closer look at their mobility behavior can offer valuable insights about the choice between these two transport modes. Knowing when and why people prefer the ICE car over the EV may reveal some of the factors that are still slowing down EV adaption. Furthermore, being able to predict this choice can be used to optimize smart charging applications or also to offer automated and personalized route planning.

In this study, we analyze a tracking dataset of 129 persons who own an ICE car and an additional (compact class) EV as second car. We control their mobility choice behavior on three types of factors: trip descriptors (e.g., length, duration or trip purpose), socio-demographic factors and spatio-temporal context data. Given the limited range of EVs and the well-known issue of range anxiety [7], we hypothesize that we can explain the mobility choice by the distance of the trip as the primary influencing factor, followed by (less important) influencing variables such as the duration of the planned trip (e.g. several hours vs. several days), the temperature (due to an increased heating demand the range of EVs is decreased in cold weather), or the weekday of the departure (e.g., on weekends, people might want to use the ICE car due to being more spacious or luxurious than the compact class EV).

We find that while these factors can indeed be used to infer the transport mode choice, their impact on the overall prediction accuracy is small, and the herein evaluated models are of comparably low explanatory power. It turns out that for most of their travels, the participants of the study chose the EVs independently of the predictors analyzed within this paper, and instead make their choice largely dependent on other contextual factors (such as traveling with the family). This is good news for everyone considering buying an electric vehicle: In practice, for most trips performed, people do not seem to prefer one of the drive technology over the other. As part of our experiments, we additionally evaluated how well our models generalize across different users. We found that the fitted models are hardly transferable to unseen users but that the knowledge about individual users greatly increases the prediction accuracy for these users. This is an additional hint that while there are only weak relations between the examined features and the mode choice, the users' mode choice behavior is unique and regular.

The rest of this paper is structured as follows: In Section 2, existing mode choice models are evaluated, in particular with respect to EVs. Section 3 introduces the dataset in more detail and explains the various preprocessing steps. The different models evaluated are presented in Section 4, and their results and predictive power in Section 5. Finally, we discuss and conclude the paper in Section 6. 


\section{Related Work}

\subsection{Transport Mode Choice}

Choices of transport modes differ widely, between individuals as well as between countries. For example, the work by Zhao et al. [37] developed a clustering-based framework to understand individuals' travel mode choice behavior in multi-modal transportation. It is shown that the users exhibit different patterns in spending time by car and EV. Buehler [5] analyzed the travel mode choice in Germany and USA using comparable travel surveys. It is reported that Germany and America have significant differences when it comes to the travel behavior of their citizens, with Germans making a four times higher share of trips by foot, bike and public transport, even though they are both developed countries and have very high motorization rates. Moreover, previous studies demonstrated that travel mode choice is influenced by various factors, including individual socio-demographic information $[32,2]$, travel characteristics (e.g. distance, duration) [6], weather conditions [17], etc.

In recent years, a series of transport mode choice models were developed based on the above-mentioned influence factors, among which logit models are one of the most widely-used forms (cf. [9, 28, 27, 24, 2, 15]). For instance, in the work by Bin Miskeen et al. [27], a binary logit model was developed to model the interurban travel mode choice behavior in Libya. Specifically, the probability of car drivers shifting to the use of buses was examined. Lee et al. [15] compared the performance of a Multinomial Logit Model (MNL) with four types of Artificial Neural Networks (ANN) for travel mode choice modeling. The results indicate that the ANN models are superior to the MNL model, however the ANN models still struggle to achieve the same level of explanatory power and robustness as the MNL model does. Although there are several studies that investigate people's transport mode choice with logit models, little attention has been paid to study influence factors on the choice between ICE car and EV. This paper builds upon a comprehensive empirical study that explores the influencing factors by modeling the choice between ICE car and EV.

\subsection{Anxiety and E-cars}

In the discussion about EV adoption, an important factor is range anxiety, i.e., the concern of (potential) users of electric cars that the restricted range of EVs will restrict their mobility options. Several studies have shown that EVs can cover much of a user's mobility needs, but not all. For instance, Pearre et al. [25] analyzed the user behavior in Atlanta, USA, and came (among other findings) to the result that 100 miles or more of daily driving occurs on average only on 23 days in the year. In addition, Woodjack et al. [34] show that users can, to some degree, adapt their travel behavior and range anxiety decreases over time. Tamor and Milacic [31] discuss in more detail why modestrange EVs in multi-vehicle households are a more cost-effective means to electrify personal travel than general-purpose EVs, not taking into account government interven- 
tions. Given that background, it is desirable to have a deeper understanding of the mobility behavior of people with access to EVs and ICE cars, which is what we want to achieve with this study.

\subsection{E-car Choice Models}

Present studies about EV choices mostly focus on the choice to buy an EV rather than the factors of the decision when to use it. Several studies analyzed the socio-demographic characteristics of EV-adopters, with mostly consistent results. Electric car use is shown to be positively associated with being male, middle-aged (30-50), being married and having a high income level and higher education. In addition to that, Simsekoglu [30] analyzed the socio-demographic characteristics of people owning a conventional car, an e-car and both a conventional and an electric car. Their finding was that the latter two have a more similar profile than sole conventional owners with one of the others.

Zhang et al. [35] examined how consumer choices are influenced by car specifications, prices and government incentives with using a Random-Coefficient Discrete Choice Model. It is found that EV technology improvements, toll waivers and charging station density have positive effects on EV demand for both private consumers and business buyers. The work by Lebeau et al. [14] explored the choice of EVs in city logistics. The results suggested several important measures, such as developing a larger charging infrastructure, implementing financial incentives through subsidies or tax exemption. Concerning governmental incentives to promote EV adoption, it has been shown that measures that reduce the purchase price for customers have the highest impact [1].

Literature that analyzes the choice of households that own an EV and an ICE car is very rare. We could identify only a single study that took place in Denmark $[10,11]$. The study took place from 2011 to 2014 and over this time, 567 households received an EV for about three months. All households were required to already own an ICE vehicle in order to participate in the study. As the study period was in the early years of EV adoption, the distributed EVs had an effective range of only about $90 \mathrm{~km} \mathrm{(150}$ $\mathrm{km}$ of advertised range). In [10], weekends, temperature, precipitation and wind speed were reported as significant factors, however, their explanatory value (e.g., effect size) was not reported. Interestingly, the trip distance was not a significant factor for the choice between transportation modes. Even though this last finding is rather surprising, it was not further discussed in [10]. The work in [10] represents only a single point of evidence where study participants are observed over a short period of time using earlyyear EVs. We plan to extend this work by investigating the explanatory value of the analyzed factors and by further investigating the influence of the trip distance. 


\section{Data}

For our empirical analysis, we used data from a large-scale pilot project that evaluated the use of a comprehensive Mobility as a Service (MaaS) package [21]. For the duration of one year, 138 Switzerland-based participants were given a battery electric vehicle (with a range marketed as $300 \mathrm{~km}$ ), a general public transport pass valid for the whole region, as well as access to several car- and bike-sharing programs. 129 of those participants previously already owned an ICE car and continued using it throughout the one-year study period, during which they also had to install a GPS-tracking app on their smartphones which recorded their mobility behavior (with a median time of $13.9 \mathrm{sec}-$ onds between two consecutive GPS positionfix recordings). Additionally, the movement of the EV and the state of the EV (including state of charge of the battery) was recorded.

The app-based tracking data was automatically segmented into so-called staypoints whenever a user is stationary (e.g., not moving out of a small radius for a non-negligible amount of time). The study participants labeled the staypoints in the app with a highlevel purpose (one of home, work, errand, leisure, wait, and unknown). We call a staypoint an activity if it has an important purpose (everything except for wait and unknown) or if its duration is longer than 25 minutes. All movements between two activities are summarized as a trip. A trip is segmented in triplegs, whereby a tripleg describes all continuous movement with the same mode of transport and without line changes (for public transport). Users labeled triplegs with a mode of transport (one of car, e-car, train, bus, tram, bicycle, e-bike, walk, and a range of less frequently used transport modes such as airplanes, boats or coaches). The app makes label suggestions for the mode of transport and the activity purpose that a user can either accept or correct.
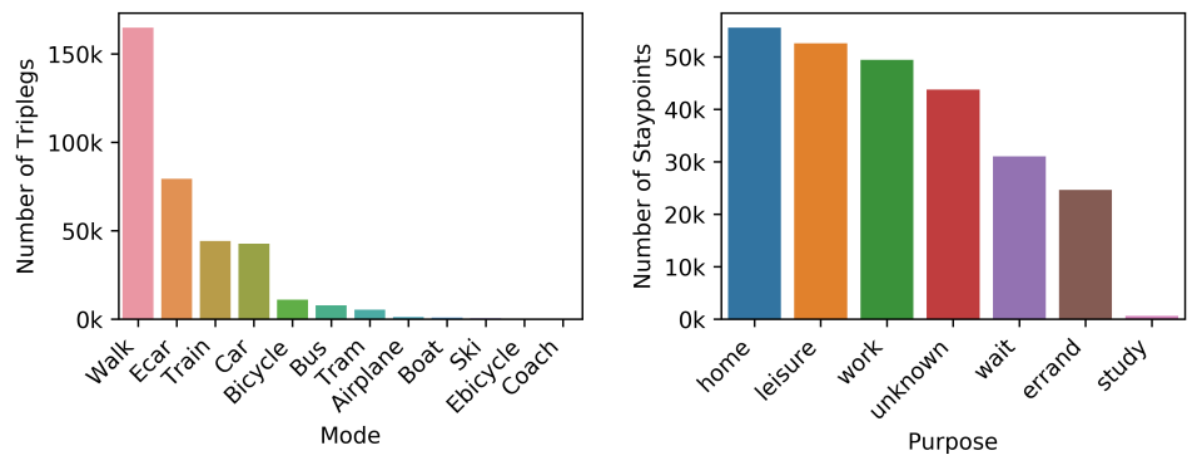

Fig. 1. Triplegs and staypoints recorded within the SBB Green Class study in 2017.

The study participants were tracked starting from November 2016 to the end of December 2017. In total, the 138 participants generated approx. 230 million GPS positionfixes, segmented into 326'926 staypoints, 242'012 trips, and 465'195 triplegs. Figure 1 shows 
the distribution of tripleg transport modes on the left and the distribution of staypoint purposes on the right. Out of all triplegs $122^{\prime} 063$ were either covered by car (42'739; $35.0 \%$ ) or e-car $\left(79^{\prime} 324 ; 65.0 \%\right)$, which resulted in a total distance of $1^{\prime} 450$ ' $401 \mathrm{~km}$ by car and 1'258' $553 \mathrm{~km}$ by e-car.

The motivation of study participants usually decreases over time. This resulted in study participants taking less care when validating the detected transport modes. In particular the distinction between car and e-car/EV is difficult from GPS data alone, which is why a careful and trustworthy validation would be of essence. As we are unable to force study participants to validate their input, we additionally use the data collected by the EV itself for validation. This data includes the start and end locations of each drive registered by the $\mathrm{EV}$, with an associated change in kilometers and state of charge (SoC). The matching of both datasets is tricky because both are subject to different spatiotemporal uncertainties. We use the rule-based approach for the matching of app-recording and EV-recording pairs described in [21]. This approach considers the spatio-temporal distance between start and end points, the temporal overlap and the ratio of the length of the app-tripleg and the difference in the km-counter of the EV. Afterwards, we checked for consistency in sequences of trips, in order to ensure that impossible combinations like driving to work by car and back by EV do not appear in the dataset.

In addition, we used weather data collected by the automatic weather monitoring network of the Swiss Federal Office of Meteorology and Climatology MeteoSuisse ${ }^{1}$. As this data is only available at certain measurement locations, it was interpolated spatially and temporally following the method outlined in [4]. In essence, a temperature correction coefficient that is dependent on the distance to the closest weather stations as well as on the difference in elevation is computed and applied to every GPS positionfix for which a temperature measurement is required (usually the start of triplegs and trips). From the automatic weather monitoring network, the temperature as well as the precipitation were extracted, as these are generally the weather factors influencing transport mode choice the most. Figure 2 shows the measurements at the beginning of each tripleg as well as their average over the year.

Finally, the study participants were asked to fill in a range of surveys. Of particular importance are the socio-demographic features recorded this way, a selection of which is shown in Table 1. It is visible from this data that there is a bias towards male participants with above average age and income. The demographic data corresponds to the study participants, however, the car can theoretically be used by anyone in the household. As not all of the participants have both an electric and an ICE car, we removed 9 people who did not own a car at the time the study started, which resulted in 129 participants whose data are used for the analysis presented below.

\footnotetext{
${ }^{1}$ For information about the automatic weather monitoring network of MeteoSuisse consider https://www.meteoschweiz.admin.ch/home/mess-und-prognosesysteme/bodenstationen/automatisches-messnetz.html.
} 

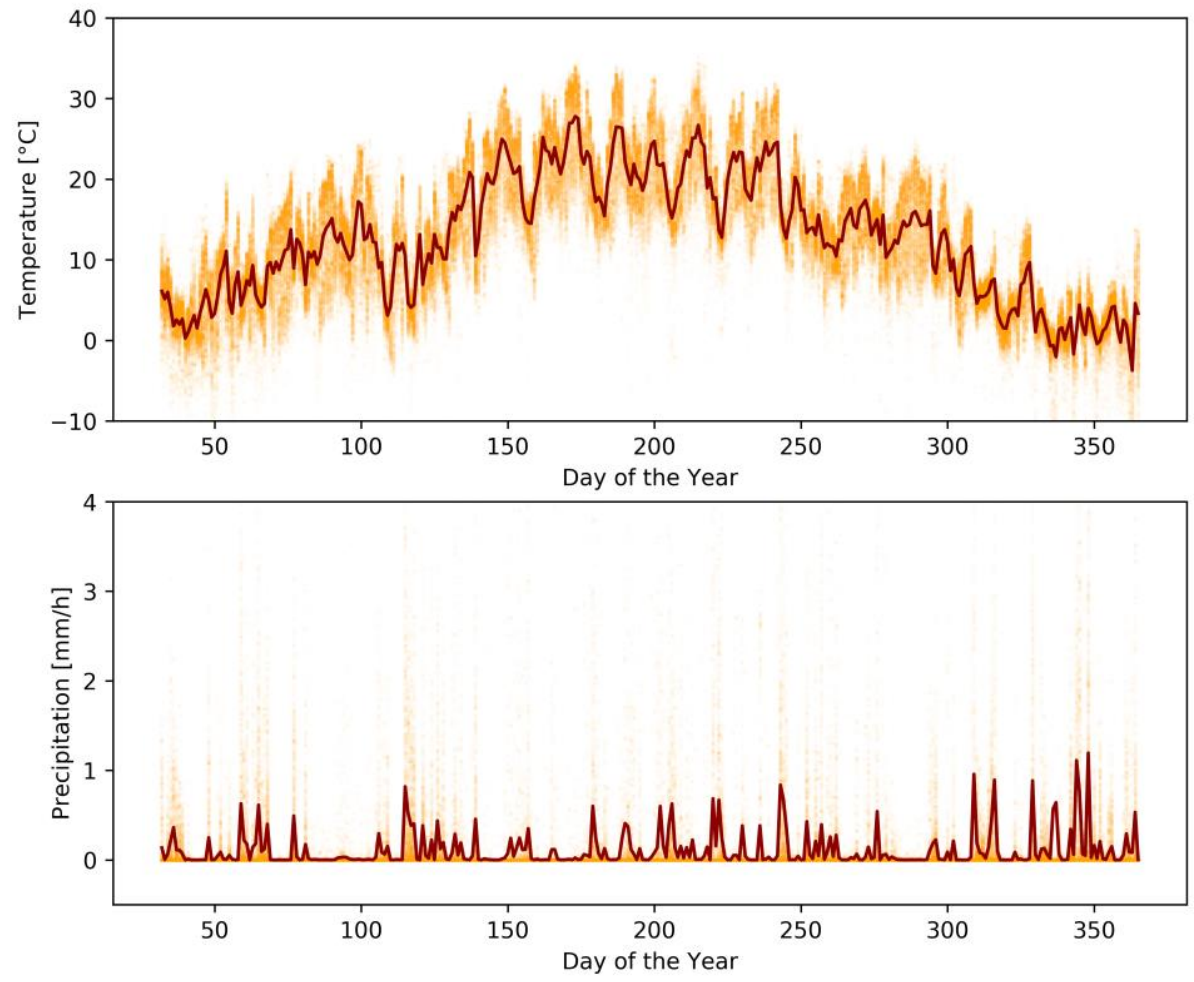

Fig. 2. Temperature and precipitation at the start of each tripleg over the course of the one-year study period as well as the average as bold line. The offset in the beginning results from a slightly delayed start of the study and the fact that participants received their EV on different dates.

Table 1. Features taken from the socio-demographic survey and used within the models presented in this paper. Note that the median income is exactly CHF $17^{\prime} 500$ as this variable was measured as one of the following classes: $\left\{6^{\prime} 500,10^{\prime} 000,13^{\prime} 500,17^{\prime} 500,22^{\prime} 500\right\}$. *Number of cars prior to receiving the EV. The 9 participants without cars are not considered in the other distributions in the table.

\begin{tabular}{lrll}
\hline Feature & \multicolumn{3}{l}{ Distribution } \\
\hline Sex & 111 & male & $(86.0 \%)$ \\
& 18 & female & $(14.0 \%)$ \\
Number of cars in household* & 9 & with 0 cars & $(6.5 \%)$ \\
& 47 & with 1 cars & $(34.1 \%)$ \\
& 67 & with 2 cars & $(48.6 \%)$ \\
12 & with 3 cars & $(8.7 \%)$ \\
3 & with 4 cars & $(2.2 \%)$ \\
\hline
\end{tabular}

AGILE: GIScience Series, 1, 2020.

Full paper Proceedings of the 23rd AGILE Conference on Geographic Information Science, 2020. Editors: Panagiotis Partsinevelos, Phaedon Kyriakidis, and Marinos Kavouras

This contribution underwent peer review based on a full paper submission. https://doi.org/10.5194/agile-giss-1-2-2020 | @ Authors 2020. CC BY 4.0 License. 


\begin{tabular}{lrll}
\hline Work status & 118 & working full-time & $(91.5 \%)$ \\
10 & working part-time & $(7.8 \%)$ \\
& 1 & not working & $(0.8 \%)$ \\
Household size (no. of people) & 11 & with 1 person & $(8.5 \%)$ \\
& 36 & with 2 persons & $(27.9 \%)$ \\
& 24 & with 3 persons & $(18.6 \%)$ \\
& 33 & with 4 persons & $(25.6 \%)$ \\
& 19 & with 5 persons & $(14.7 \%)$ \\
& 6 & with 6+ persons & $(4.7 \%)$ \\
Age & 47.3 & \pm 7.6 years & \\
Household income & $16^{\prime} 791$ & \pm CHF 4'358 \\
& & (median CHF 17'500) \\
Total & 129 & eligible participants & \\
\hline
\end{tabular}

\section{Method}

In our work, we analyze the users' mobility choice behavior on two levels: The atomic tripleg and the aggregated tour level. We choose to analyze the mobility behavior on the tripleg level because it is the smallest unit in which we measure movement. However, it is likely that choices between taking the ICE car and the EV cannot be explained solely on a tripleg level. This becomes clear if we look at the example of a user that leaves home in the morning to go to work, goes shopping after work, and then returns home. If such users choose to go to work using the EV, they will be forced to also take the EV in the evening for their errands and the way home. Looking at complete tours allows us to more closely follow the decision process of a user, who always has to take the complete mobility chain until reaching the initial position again into account. Even if both perspectives are different, we expect that there will be a large overlap between the mode choice models on the tripleg level and the tour level model.

\subsection{Data Preprocessing}

Based on the staypoints and triplegs provided by the tracking app, we use a hierarchical model of human mobility to extract trips (explained in Section 3) and tours. A tour is defined as a closed loop in the daily mobility of a user. This means we add all triplegs to the same tour that fulfill all of the following requirements:

- End respectively start less than 350 meters apart from each other.

- Were undertaken on the same day ( \pm 12 hours).

- Are longer than 350 meters. 
All tours that start and end at the user's home location are called home-based tour or journey. In general, it is possible for tours to contain sub-tours. An example for a typical journey that contains a sub-tour is the following: A person wakes up at home, takes the car to go to work, walks to a local grocery store during her break to buy snacks and some groceries, then goes back to work and drives home after she is finished. Here, all trips undertaken are part of the user's journey and the trip to the supermarket and back is a sub-tour. Our method is implemented such that also sub-tours are detected. For our analysis, we consider all tours that contained either a minimum of one tripleg covered by car or e-car and start and end at a user's home (i.e., all journeys).

\subsection{Outlier Removal and Downsampling of the Majority Class}

As the dataset is from a smartphone-based tracking study it is subject to noise and uncertainty. This can include sudden jumps, positions that are frozen over a longer period of time or gaps. We filter these tracking-related outliers by excluding triplegs that have an abnormally high average speed or abnormally low average speed ${ }^{2}$ and in a second step, we fit a robust 2-dimensional normal distribution to the length-duration distribution of the triplegs and exclude the least-likely $3 \%$ of the data.

The final data after filtering includes 39'409 car triplegs, 73'388 e-car triplegs, 9'339 car tours and 24'550 e-car tours. To avoid bias when reporting results, we randomly sample from the majority class without replacement such that the tour and tripleg dataset are class-balanced with respect to the transport mode (car or e-car). This leads to a new dataset of $78^{\prime} 818$ class-balanced triplegs and 18'678 class-balanced tours.

\subsection{Feature Engineering and Extraction}

To analyze the choice of a specific user, we test variables from three different categories: socio-demographic variables sourced from user surveys, tour respectively tripleg descriptors recorded by the tracking app (and enhanced by the EV data) and spatiotemporal context variables created by combining the available data with other environmental datasets, most notably the temperature and precipitation.

Table 2 shows all the features that we used as predictors in the models for the tripleg and the tour based approaches. As not all the data is available on the tour level (in particular, only the aggregate of all triplegs can be considered when modeling mode choice on a tour level), the predictors resp. models slightly differ. The features shown in Table 2 are the features that were originally generated, however, many of them are only marginally useful as predictors, and thus not always reported anymore. The tripleg length is computed based on a map-matched geometry, i.e., all positionfixes making up

\footnotetext{
${ }^{2}$ We consider an average speed of more than $220 \mathrm{~km} / \mathrm{h}$ and less than $3 \mathrm{~km} / \mathrm{h}$ as abnormal.
} 
a tripleg were matched to the respective transport network using the map matching algorithm available through the Open Source Routing Machine (OSRM) $[18]^{3}$. The purpose of a trip is the next activity's purpose, while the purpose of a tour is computed using a majority vote on the purposes of all the staypoints visited during the tour. For the spatio-temporal context, we always considered the starting time of the tripleg resp. tour (e.g., the temperature is simply taken at the location and time of the first positionfix of the respective tripleg or tour). The socio-demographic features were attached to each tripleg resp. tour based on the user ID and used as independent variable similar to the tripleg/tour descriptors and spatio-temporal context.

Table 2. The different features used for this study and the respective variable's type. Nominal variables are encoded as dummy variables, each denoting the presence of one of the labels. *For tour-level analyses these features are not used. **Prior to the start of the study. $* * *$ Normalized to lie in $[0,1]$.

\begin{tabular}{|c|c|c|}
\hline Feature & Type & Coding \\
\hline \multicolumn{3}{|l|}{ Tripleg/Tour descriptors } \\
\hline Tripleg length* & Ratio & {$[0, \infty)$} \\
\hline Tripleg duration* & Ratio & {$[0, \infty)$} \\
\hline $\begin{array}{l}\text { Length of all triplegs in tour cov- } \\
\text { ered either with car or } e \text {-car }\end{array}$ & Ratio & {$[0, \infty)$} \\
\hline Tour length & Ratio & {$[0, \infty)$} \\
\hline Tour duration & Ratio & {$[0, \infty)$} \\
\hline Previous staypoint duration* & Ratio & {$[0, \infty)$} \\
\hline Next staypoint duration* & Ratio & {$[0, \infty)$} \\
\hline Purpose of trip* & Nominal & $\begin{array}{l}\text { \{home, work, errand, leisure, } \\
\text { wait, unknown }\}\end{array}$ \\
\hline Purpose of tour & Nominal & $\begin{array}{l}\text { \{home, work, errand, leisure, } \\
\text { wait, unknown }\}\end{array}$ \\
\hline \multicolumn{3}{|l|}{ Socio-demographic data } \\
\hline Sex & Nominal & $\{$ male, female $\}$ \\
\hline Age & Ratio & {$[0, \infty)$} \\
\hline Cars in household** & Ratio & {$[0, \infty)$} \\
\hline Employment status & Nominal & $\{$ working, not working $\}$ \\
\hline Household income & Ratio & {$[0, \infty)$} \\
\hline
\end{tabular}

${ }^{3}$ The OSRM can be downloaded from http://project-osrm.org. 


\begin{tabular}{cll}
\hline $\begin{array}{c}\text { Household size } \\
\text { Spatio-temporal context }\end{array}$ & Ratio & {$[0, \infty)$} \\
\hline Hour of day*** & Ordinal & $\{0,1, \ldots, 23\}$ \\
Weekday/weekend & Nominal & $\{$ weekday, weekend $\}$ \\
Month of year*** & Ordinal & $\{1, \ldots, 12\}$ \\
Temperature at start & Interval & {$[-273.15, \infty)$} \\
Precipitation at start & Ratio & {$[0, \infty)$} \\
\hline
\end{tabular}

Some of the values for the features were missing and were imputed using the median of all available data for this variable. For triplegs, $4 \%(5,328)$ of the values for sex, age, cars in household, employment status and household size (1 user), and 15\% (17’209) of the values for household income were missing. As the tours are based on the same data, they show a similar pattern of missing data. Finally, all the nominal predictors having more than two classes were encoded using dummy variables. This means that instead of having one feature that denotes the purpose, we instead have six features that indicate if the purpose was one of the given six categories in a binary fashion (one-hot encoding).

\subsection{Modeling E-Car Choice}

To determine the suitability of the introduced features for predicting the mode choice, we run the following 4 experiments for the tripleg and the tour level.

Random Forest Model. In the first experiment, we fit a random forest (RF) classifier on all features to predict the choice of all users. RFs are a class of ensemble models that combine several decision trees with random feature selection on bootstrapped samples (a variation of bagging with decision trees). RFs were introduced in [3] and are robust against overfitting and require little hyperparameter tuning. A RF is a non-linear classifier that usually performs close to the state-of-the-art on tabular data for regression and classification problems. However, while the visualization of decision boundaries and the variable importance offer some degree of explainability for RF-models, it is hard to understand the exact relationship between inputs and the target. We therefore use the RF model to create an upper-boundary baseline for the accuracy and fit logistic regression based mode-choice models for interpretation.

Logit Model. To get a better and more interpretable understanding of the relationship between input variables and the decision of the user to take the ICE car or the EV, we fit a logarithmic regression model (logit). Logits are the widely accepted standard in transport planning for mode-choice models (cf. [9]), especially because of their capability to work with mixed integer and continuous input variables and their interpretability. However, due to their linear nature, all non-linear and all cross-relations between 
inputs and the target have to be precalculated explicitly. We define the logistic regression model that predicts the probability of the target variable being 1 as

$$
p_{l r}\left(x_{i}\right)=\frac{1}{1+e^{-x_{i}^{T} \beta}}
$$

where $x_{i}$ is the $i^{\text {th }}$ vector of observations and $\beta$ are the weights of the model. We furthermore define the logistic per-sample loss as

$$
L_{\text {sample }}=-y_{i} \log \left(p_{l r}\left(x_{i}\right)\right)-\left(1-y_{i}\right) \log \left(1-p_{l r}\left(x_{i}\right)\right)
$$

where $y_{i}$ is the true binary label associated with the $i^{\text {th }}$ observation. We identify the parameters $\beta$ by minimizing the following regularized total loss function

$$
L_{\text {ridge }}=\sum_{i=1}^{n} L_{\text {sample }}\left(x_{i}, y_{i}\right)+\lambda \sum_{j=1}^{m} \beta_{j}^{2}
$$

where $\alpha$ is a tuneable hyperparamter, $n$ is the number of data points, $\beta_{j}$ is the $j^{\text {th }}$ model parameter and $m$ is the number of model parameters. The predictions $p_{l r}\left(x_{i}\right)$ are used to create the following classifier:

$$
f\left(x_{i}\right)=\left\{\begin{array}{lll}
0 & \text { if } & p_{l r}\left(x_{i}\right)<0.5 \\
1 & \text { if } & p_{l r}\left(x_{i}\right) \geq 0.5
\end{array}\right.
$$

To make up for the linearity of the logit model, we manually transform some features based on their histograms. In particular, if a feature shows a periodic pattern (e.g., the temperature over the course of a year) or follows a power law (e.g., the distance travelled), we apply a trigonometric resp. a logarithmic function. The resulting transformed features are directly used as input for the logit model.

User Specific Models. In the final experiment, we want to investigate the generalization properties of the random forest and the logit models for the given problem. We refit the same RF and logit models but use a user-specific data split for the cross-validation scheme.

This means that the set of users in the training data and the set of users in the test data are disjunct. This test allows us to analyze if the random forest model could actually identify underlying user-independent rules that explain under which circumstances people prefer to take an EV or an ICE car. If the random forest fails to generalize in this experiment, it indicates that it simply remembers user-specific patterns and preferences.

\subsection{Model Performance Metrics}

We use two metrics to evaluate the performance of the proposed models: Accuracy and $R^{2}$. Accuracy is a widely accepted and easy to interpret classification metric that is defined as 


$$
a c c=\frac{y_{\text {correct }}}{y_{\text {correct }}+y_{\text {incorrect }}}
$$

where $y_{\text {correct }}$ and $y_{\text {incorrect }}$ are the counts of correctly and incorrectly classified samples, respectively. Accuracy is very easy to interpret but it is misleading if class counts in the sample are unbalanced. As we operate in a binary classification regime, $50 \%$ accuracy corresponds to random guessing.

As a second metric, we report the McFadden (pseudo) $R^{2}$ introduced in [23]. This score represents an alternative to the well-known $R^{2}$ score which is not meaningful for binary classification tasks. The McFadden pseudo $R^{2}$ is defined as

$$
R_{\text {McFadden }}^{2}=1-\frac{\ln L_{1}}{\ln L_{0}}
$$

where $L_{0}$ is the likelihood of the null model (a model only fitted with the intercept) and $L_{1}$ is the likelihood of the controlled model. As the logarithm of the likelihood is always negative, the log-likelihood of a good model goes towards 0 and because $L_{0}$ is constant, this drives the McFadden score towards 1. The other way around, if the analyzed model's log-likelihood is similar to the one of the null model (this means without great explanatory value), then the McFadden pseudo $R^{2}$ score goes towards 0 . While this behavior is similar to the regular $R^{2}$, the values of the McFadden pseudo $R^{2}$ are usually significantly lower, such that a score above 0.2 is already considered good [22]. We calculate the McFadden pseudo $R^{2}$ score according to Equation 6 using a logit model only containing an intercept as the null model. This means the fitted model is able to only predict a single constant for all samples. The logit model is fitted without any regularization and using the Limited-memory BFGS (cf. [20]) solver.

\section{$5 \quad$ Results}

In the following, we describe the fitting of the final models used for each of the experiments and report their accuracy, pseudo $R^{2}$ and the significance and importance of various predictors. Furthermore, we will quickly elaborate on the most important takeaways for each model. A comparative discussion of the different models and the implications on the mode choice problem is given in Section 6. All the data preprocessing and all the models were implemented in Python using the scikit-learn [26] (for the random forests) and statsmodels [29] (for the logistic regression) libraries.

\subsection{Random Forest Models}

The random forest baselines for the tour and the tripleg level experiment simply use all features introduced above to predict the binary outcome $\{$ car, $e$-car $\}$. For both models, we chose 1000 trees, the Gini impurity as quality criterion, the square root of the total number of features as the number of features per split and an unrestricted tree depth as hyperparameters. The data was randomly split according to a 3:1 proportion; while the 
first $75 \%$ were used to train the RF, the remaining $25 \%$ were used to compute the accuracy of the prediction (cf. Section 4.5).

Table 3. Accuracies and pseudo $R^{2}$ for the models used within this paper.

\begin{tabular}{lrr}
\hline Model & Accuracy & Pseudo $R^{2}$ \\
\hline Random Forest Triplegs & $78.86 \%$ & 0.3060 \\
Random Forest Tours & $74.04 \%$ & 0.2198 \\
Logit Triplegs & $59.00 \%$ & 0.0195 \\
Logit Tours & $58.46 \%$ & 0.0328 \\
Random Forest Triplegs (User Split) & $65.40 \%$ & 0.1032 \\
Random Forest Tours (User Split) & $59.96 \%$ & 0.0273 \\
Logit Triplegs (User Split) & $57.28 \%$ & 0.0055 \\
Logit Tours (User Split) & $52.43 \%$ & -0.0072 \\
\hline
\end{tabular}

Table 3 shows the values for accuracy and the pseudo $R^{2}$ for the different models. Figure 3 contains the same information, visualized for a more immediate comparison of the models. The baseline RF reaches an accuracy of $78.86 \%$ on the tripleg level, and $74.04 \%$ on the tour level, indicating that it is feasible to predict if someone chooses the ICE car or the e-car. Similarly, the high pseudo $R^{2}$ values of 0.3060 resp. 0.2198 indicate that the predictors (in their aggregation) are able to explain the variance in transport mode choice well.

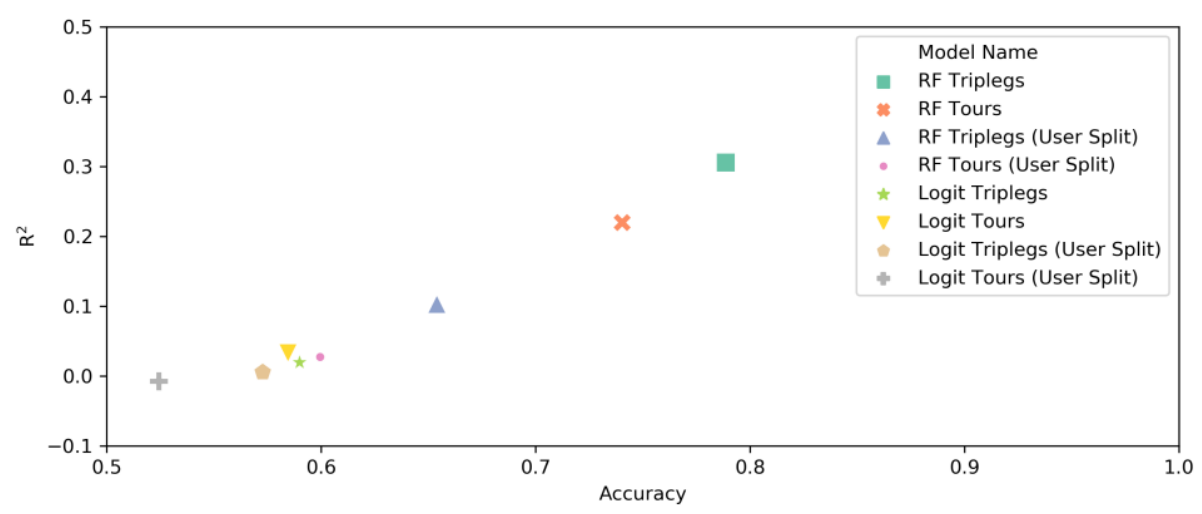

Fig. 3. Accuracies vs. pseudo $R^{2}$ and feature importances of all the models evaluated within this work. 


\subsection{Linear Logistic Regression Models}

To have a more readily interpretable model, we fitted a logit model (only considering linear superpositions) between the features and the discrete target. The here presented models for triplegs and tours are the result of both a manual search for a model that balances explainability and prediction ability as well as an automated model finding process.

Mode Choice on a Tripleg Level. Table 4 shows the chosen predictors for the logit model on the tripleg level, and their model parameters and significances. The omitted predictors (compared to all the features displayed in Table 2) were either not significant given the training data, or did not substantially add to the model. It can be seen that the presence of the long-distance marker (triplegs longer than $100 \mathrm{~km}$ ) strongly drives the choice, as do the distance and duration related predictors as well as the weekday/weekend marker. Note that since the models are not normalized (except the hour of day and month of year features, cf. Table 2), the coefficients cannot be directly used to compare the importance of different predictors. However, having these non-normalized coefficients allows for example comparing the influence of the difference in $1^{\circ}$ Celsius with a distance increase of $10 \mathrm{~km}$. From the model, it can be seen that the weather context is only minimally important, as is the age, household size and hour of day.

Table 4. Model parameters and predictor significances for the tripleg logit model. *Significant at the $\mathrm{p}<0.05$ level.

\begin{tabular}{lrrrr}
\hline Feature & Coefficient & Std. Error & $z$-Value & $p$-Value \\
\hline Intercept & 3.7693 & 0.310 & 12.175 & $0.000^{*}$ \\
Weekday/weekend & -0.4806 & 0.017 & -27.879 & $0.000^{*}$ \\
Temperature & 0.0078 & 0.001 & 7.719 & $0.000^{*}$ \\
Precipitation & 0.0394 & 0.012 & 3.244 & $0.001^{*}$ \\
Sex & -0.1872 & 0.025 & -7.638 & $0.000^{*}$ \\
Age & 0.0086 & 0.001 & 8.037 & $0.000^{*}$ \\
Numbers of cars in household & -0.0636 & 0.012 & -5.425 & $0.000^{*}$ \\
Work status & 0.3381 & 0.023 & 14.635 & $0.000^{*}$ \\
Household size & 0.0689 & 0.006 & 11.811 & $0.000^{*}$ \\
Long-distance tripleg & -1.7695 & 0.064 & -27.848 & $0.000^{*}$ \\
$\log$ (duration of next activity) & 0.0776 & 0.005 & 14.868 & $0.000^{*}$ \\
$\log$ (duration of prev. activity) & 0.0852 & 0.005 & 15.984 & $0.000^{*}$ \\
$\log$ (duration of tripleg) & -0.3136 & 0.018 & -17.038 & $0.000^{*}$ \\
$\log$ (length of tripleg) & 0.2011 & 0.014 & 14.614 & $0.000^{*}$ \\
\hline
\end{tabular}




\begin{tabular}{lrrrr}
\hline $\log$ (household income) & -0.4286 & 0.031 & -14.023 & $0.000^{*}$ \\
$\sin ($ hour of day) & 0.0928 & 0.011 & 8.359 & $0.000^{*}$ \\
$\sin ($ month of year) & 0.2385 & 0.011 & 21.001 & $0.000^{*}$ \\
\hline
\end{tabular}

The logit model is greatly outperformed by the corresponding random forest. With an accuracy of $59 \%$ and significantly lower pseudo $R^{2}$ values the logit model is clearly the less powerful model. This is an indication that many of the relations that the RF model learned from the randomly split data are non-linear and not included in our self-generated more obvious set of non-linear features.

Mode Choice on a Tour Level. Looking at the logit model on the tour level (shown in Table 5), we see a similar distribution of predictor coefficients. Again, the presence of the long-distance marker has a substantial influence, just as the other distance-related predictors do. It seems that the overall length of the tour is a more important criterion than the parts covered by car resp. e-car. Again, and also indicated by the p-values, there is no significant relation between contextual factors such as the temperature, precipitation, or household characteristics. It needs to be noted that the interpretation of the impact of sex and work status on the mode choice is difficult as there is a large bias towards working males in our dataset. The accuracy of the model similarly drops to around $58.5 \%$, with a corresponding $R^{2}$ score of 0.0328 , indicating that the model is of lower explanatory power than the corresponding random forest model.

Table 5. Model parameters and predictor significances for the tour logit model. *Significant at the $\mathrm{p}<0.05$ level.

\begin{tabular}{lrrrr}
\hline Feature & Coefficient & Std. Error & $z$-Value & $p$-Value \\
\hline Intercept & -1.7069 & 0.611 & -2.792 & $0.005^{*}$ \\
Weekday/weekend & -0.4440 & 0.035 & -12.632 & $0.000^{*}$ \\
Temperature & -0.0018 & 0.002 & -0.850 & 0.395 \\
Precipitation & -0.0357 & 0.029 & -1.238 & 0.216 \\
Sex & -0.1451 & 0.049 & -2.975 & $0.003^{*}$ \\
Age & 0.0078 & 0.002 & 3.503 & $0.000^{*}$ \\
Numbers of cars in household & -0.0250 & 0.024 & -1.030 & 0.303 \\
Work status & 0.6985 & 0.260 & 2.689 & $0.007^{*}$ \\
Household size & 0.0900 & 0.012 & 7.509 & $0.000^{*}$ \\
Long-distance tour & -0.5116 & 0.053 & -9.670 & $0.000^{*}$ \\
log(length of all triplegs by car & 0.0464 & 0.009 & 5.206 & $0.000^{*}$ \\
or e-car) & & & & \\
\hline
\end{tabular}

AGILE: GIScience Series, 1, 2020.

Full paper Proceedings of the 23rd AGILE Conference on Geographic Information Science, 2020. Editors: Panagiotis Partsinevelos, Phaedon Kyriakidis, and Marinos Kavouras

This contribution underwent peer review based on a full paper submission. https://doi.org/10.5194/agile-giss-1-2-2020 | (c) Authors 2020. CC BY 4.0 License. 


\begin{tabular}{lrrrr}
\hline $\log$ (length of tour) & 0.1006 & 0.020 & 5.066 & $0.000^{*}$ \\
$\log$ (duration of tour) & 0.0620 & 0.017 & 3.704 & $0.000^{*}$ \\
$\log$ (household income) & -0.0584 & 0.062 & -0.940 & 0.347 \\
$\sin$ (hour of day) & 0.1443 & 0.024 & 6.043 & $0.000^{*}$ \\
$\sin$ (month of year) & 0.1223 & 0.023 & 5.282 & $0.000^{*}$ \\
\hline
\end{tabular}

\subsection{Random Forest - User Split}

As random forests are very robust non-linear models, they are prone to learn "hidden" connections in the data that lead to non-generalizable results. To test for these effects, we here present the results from applying the same RF model to the tripleg and tour data, but restricting training to a subset of users, and testing on another.

Looking at the reported values in Table 3 (comparing the entries for Random Forest and Random Forest (User Split)), it is easily visible that the RF model does not generalize well across users. Making predictions about unknown users lets the accuracy of the random forest drop by about $15 \%$ in both cases. The pseudo $R^{2}$ shows a similar decrease, meaning that the explanatory value of the user-independent model drops significantly. Considering that a random prediction would already yield an accuracy of $50 \%$ in the given case, the resulting accuracy of about $60 \%$ is only a slight improvement. This performance drop is evidence that the random forest trained using a random data split is remembering the users' decisions instead of identifying underlying general rules.
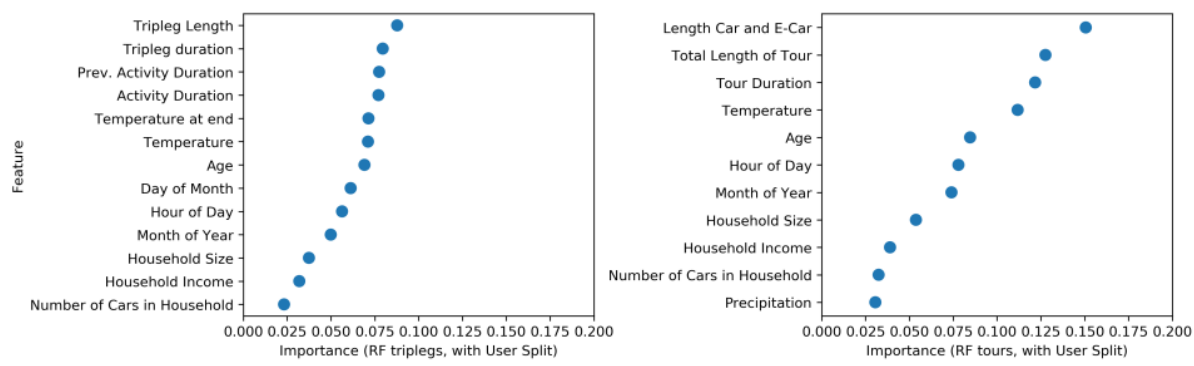

Fig. 4. Feature importances in the random forest model including a user split. On the left side, the importances for the tripleg model are shown, on the right side the ones for the tour model.

Figure 4 shows the feature importances extracted from the RF model. Similar to the logit models, the length and duration features are most important for the mode choice. This shows that the same features can be used to identify (weak) generalizable rules as well as identifying the user's unique (e.g., non-generalizable) mobility behavior. 


\subsection{Linear Logistic Regression Models - User Split}

Finally, we included the results of a logistic regression model trained on a subset of users and evaluated (for accuracy) on another subset of users. As expected, the results in Table 3 show that the original logit model already generalizes well, but thus also has a substantially lower explanatory power. We do not report the complete models here, as they do not substantially differ from the ones in Tables 4 and 5.

\section{Discussion and Conclusion}

Driven by goals of sustainability, related monetary incentives and technological advances, people buying a new car today have to decide between an electric vehicle and a regular internal combustion engine car. For many, this poses a difficult decision as they are usually not aware of how the limited range, refueling capacity and differing storage and driving characteristics might influence their future driving behavior. Using a unique constellation of people that have both types of cars at their service and have to take the decision whether to take the EV or the ICE car every day, we try to give insight into the decision process that leads to people choosing one type of car over the other. We analyze data from 129 ICE car and EV owners of a real-life study (involving 138 participants in total) that were tracked over the duration of a year via an app on their phone and via the EV itself. The chosen measures of interest are the accuracy in predicting the transport mode of previously unseen triplegs and tours and the $R^{2}$ which summarizes the explanatory power of the models. To have both a well-performing upper bound as well as more easily interpretable models, we fit random forests and logit models to features extracted from the raw data. These features are chosen with respect to the uncertainties related to EVs, namely the distance and duration of trips, the weather, personal background, and more.

Looking at the presented results in detail, there are several interesting takeaways. Even though all models are better than a random guess, their predictive power is comparably low. Only the random forest that is trained on data from all the users manages to reach both a high accuracy as well as a high $R^{2}$ score. This is a strong indication that the random forest simply learns to identify individual users by unique combinations of features, and thus also that for each user individually most mode choices follow a systematic pattern (resp. on the same trips, e.g., going to work, a single user will in most cases choose the same mode of transport). Training the models on a subset of people confirms the hypothesis that the logit models generalize as well as can be expected, namely in a similar range as the random forests on the user-split data. Looking at the predictors, we can see that the distance and duration, but also if the tripleg or tour took place on the weekend influence the choice more than factors such as the household size or age of the participant. However, contrary to our initial assumption and hypothesis, all predictors are of a weak nature, i.e., while they manage to positively influence the predictions, the resulting accuracies are still only slightly better than a random guess. A potential reason for this might be that the choice between ICE car and EV is mainly driven by variables not measured as part of the study: the size of the cars (which we 
only know for the EV), the number of people participating in a trip, the necessities of other household members, or the overall distribution of work and household duties within a family. Clustering people into groups based on these variables, or also based on the number of cars or people in a household could reduce the variability in the (subdatasets, thus increasing the predictive power of the presented models and explain better why someone chooses the ICE car or EV in a given situation. It is similarly interesting that the prediction of the transport mode choice for individual triplegs seems easier than for complete tours. We suspect that this is due to the fact that there are more triplegs that appear in a very similar fashion in the dataset (e.g., a single home-workhome tour results in two triplegs that exhibit almost the same characteristics: one from home to work, and another one back). As such, we have to consider the lower predictive power of the tour level models as a "realistic" view on how a person would choose the transport mode in a real situation.

Comparing our research to previous studies, it is in line considering the importance of individual features. However, our findings of comparatively low predictive power of the different models indicate that looking at a general population, their choices are not easily predictable and are determined by features that cannot be captured by GPS tracking or simple socio-demographic surveys alone. Considering all this, our work provides an addition to the scarce literature on car and e-car choice models, but should be extended with more in-depth analyses of additional transport mode choice predictors in the future. Similarly, our focus was limited to choice predictions based on individual data: Future work should capture the complexity of mobility choices in a more holistic way, e.g., by closely observing other household members as well. For individual drivers, it seems that they will not have to worry too much about the limited range or charging infrastructure, though, as the choice for one mode or the other is only marginally explained by the distance covered.

\section{Acknowledgements}

This research was supported by the Swiss Data Science Center (SDSC) and by the Swiss Innovation Agency Innosuisse within the Swiss Competence Center for Energy Research (SCCER) Mobility.

\section{Bibliography}

[1] Kristin Ystmark Bjerkan, Tom E Nørbech, and Marianne Elvsaas Nordtømme. Incentives for promoting battery electric vehicle (bev) adoption in norway. Transportation Research Part D: Transport and Environment, 43:169-180, 2016.

[2] Lars Böcker, Patrick van Amen, and Marco Helbich. Elderly travel frequencies and transport mode choices in greater rotterdam, the netherlands. Transportation, 44(4):831-852, 2017. 
[3] Leo Breiman. Random Forests. Machine Learning, 45(1):5-32, October 2001.

[4] Dominik Bucher, René Buffat, Andreas Froemelt, and Martin Raubal. Energy and greenhouse gas emission reduction potentials resulting from different commuter electric bicycle adoption scenarios in switzerland. Renewable and Sustainable Energy Reviews, 114:109298, 2019.

[5] Ralph Buehler. Determinants of transport mode choice: a comparison of germany and the usa. Journal of transport geography, 19(4):644-657, 2011.

[6] Naznin Sultana Daisy, Hugh Millward, and Lei Liu. Trip chaining and tour mode choice of non-workers grouped by daily activity patterns. Journal of Transport Geography, 69:150-162, 2018.

[7] Thomas Franke and Josef F Krems. Interacting with limited mobility re-sources: Psychological range levels in electric vehicle use. Transportation Research Part A: Policy and Practice, 48:109-122, 2013.

[8] Edgar G Hertwich and Glen P Peters. Carbon footprint of nations: A global, tradelinked analysis. Environmental science \& technology, 43(16):6414-6420, 2009.

[9] Stephane Hess. Advanced discrete choice models with applications to transport demand. PhD thesis, University of London, 2005.

[10] Anders F. Jensen and Stefan L. Mabit. Modelling Real Choices Between Conventional and Electric Cars for Home-based Journeys. In The 14th International Conference on Travel Behaviour Research (IATBR), pages19-23, 2015.

[11] Anders Fjendbo Jensen, Thomas Kjær Rasmussen, and Carlo Giacomo Prato. A Joint Route Choice Model for Electric and Conventional Car Users. In International Choice Modeling Conference (ICMC), 2017.

[12] George Kamiya, Jonn Axsen, and Curran Crawford. Modeling the ghg emissions intensity of plug-in electric vehicles using short-term and long-term perspectives. Transportation Research Part D: Transport and Environment, 69:209-223, 2019.

[13] Christian Andreas Klöckner, Alim Nayum, and Mehmet Mehmetoglu. Positive and negative spillover effects from electric car purchase to car use. Transportation Research Part D: Transport and Environment, 21:32-38,2013.

[14] Philippe Lebeau, Cathy Macharis, and Joeri Van Mierlo. Exploring the choice of battery electric vehicles in city logistics: A conjoint-based choice analysis. Transportation Research Part E: Logistics and Transportation Review, 91:245-258, 2016. 
[15] Dongwoo Lee, Sybil Derrible, and Francisco Camara Pereira. Comparison of four types of artificial neural network and a multinomial logitmodel for travel mode choice modeling. Transportation Research Record, 2672(49):101-112, 2018.

[16] Fangyi Li, Bofeng Cai, Zhaoyang Ye, Zheng Wang, Wei Zhang, Pan Zhou, and Jian Chen. Changing patterns and determinants of transportation carbon emissions in chinese cities. Energy, 174:562-575, 2019.

[17] Chengxi Liu, Yusak O Susilo, and Anders Karlström. The influence of weather characteristics variability on individual's travel mode choice in different seasons and regions in sweden. Transport Policy, 41:147-158, 2015.

[18] Dennis Luxen and Christian Vetter. Real-time routing with openstreetmap data. In Proceedings of the 19th ACM SIGSPATIAL International Conference on Advances in Geographic Information Systems, GIS '11, pages 513-516, New York, NY, USA, 2011. ACM.

[19] Jennifer MacDonald. Electric vehicles to be 35\% of global new car sales by 2040. Bloomberg New Energy Finance, 25, 2016.

[20] Robert Malouf. A comparison of algorithms for maximum entropy parameter estimation. In proceedings of the 6th conference on Natural language learning - Volume 20, pages 1-7. Association for Computational Linguistics, 2002.

[21] Henry Martin, Henrik Becker, Dominik Bucher, David Jonietz, Martin Raubal, and Kay W Axhausen. Begleitstudie sbb green class - abschlussbericht. Arbeitsberichte Verkehrs- und Raumplanung, 1439, 2019.

[22] D McFadden. Quantitative methods for analyzing travel behaviour of individuals: Some recent developments (cowles foundation discussion papers no. 474). Cowles Foundation for Research in Economics, Yale University, 1977.

[23] Daniel McFadden et al. Conditional logit analysis of qualitative choice behavior. 1973.

[24] Marcel Paulssen, Dirk Temme, Akshay Vij, and Joan L Walker. Values, attitudes and travel behavior: a hierarchical latent variable mixed logit model of travel mode choice. Transportation, 41(4):873-888, 2014.

[25] Nathaniel S Pearre, Willett Kempton, Randall L Guensler, and Vetri VElango. Electric vehicles: How much range is required for a day's driving? Transportation Research Part C: Emerging Technologies, 19(6):1171-1184, 2011. 
[26] F. Pedregosa, G. Varoquaux, A. Gramfort, V. Michel, B. Thirion, O. Grisel, M. Blondel, P. Prettenhofer, R. Weiss, V. Dubourg, J. Vanderplas, A. Passos, D. Cournapeau, M. Brucher, M. Perrot, and E. Duchesnay. Scikit-learn: Machine learning in Python. Journal of Machine Learning Research, 12:2825-2830, 2011.

[27] Manssour A. A. Bin Miskeen, Ahmed M. Alhodairi and Riza A. A. Bin O. K. Rahmat. Modeling of intercity transport mode choice behavior in libya: a binary logit model for business trips by private car and intercity bus. Australian Journal of Basic and Applied Sciences, 7(1):302-311, 2013.

[28] Muhammad Isran Ramli, Y Ooeda, and T Sumi. Study on mode choice model of trip for daily household logistic based on binomial logit model. In Proceeding The 3th International Conference of Transportation and Logistic (T-LOG), Fukuoka, September, 2010.

[29] Skipper Seabold and Josef Perktold. Statsmodels: Econometric and statistical modeling with python. In 9th Python in Science Conference, 2010.

[30] Özlem Simsekoglu. Socio-demographic characteristics, psychological factors and knowledge related to electric car use: A comparison between electric and conventional car drivers. Transport Policy, 72:180-186, 2018.

[31] Michael A Tamor and Milos Milacic. Electric vehicles in multi-vehicle households. Transportation Research Part C: Emerging Technologies, 56:52-60, 2015.

[32] Akshay Vij, André Carrel, and Joan L Walker. Incorporating the influence of latent modal preferences on travel mode choice behavior. Transportation Research Part A: Policy and Practice, 54:164-178, 2013.

[33] Rashid A Waraich, Matthias D Galus, Christoph Dobler, Michael Balmer, Göran Andersson, and Kay W Axhausen. Plug-in hybrid electric vehicles and smart grids: Investigations based on a microsimulation. Transportation Research Part C: Emerging Technologies, 28:74-86, 2013.

[34] Justin Woodjack, Dahlia Garas, Andy Lentz, Thomas Turrentine, Gil Tal, and Michael Nicholas. Consumer perceptions and use of driving distance of electric vehicles: Changes over time through lifestyle learning process. Transportation Research Record, 2287(1):1-8, 2012.

[35] Yingjie Zhang, Zhen Sean Qian, Frances Sprei, and Beibei Li. The impact of car specifications, prices and incentives for battery electric vehicles in norway: Choices of heterogeneous consumers. Transportation Research Part C: Emerging Technologies, 69:386-401, 2016. 
[36] Yue-Jun Zhang, Zhao Liu, Chang-Xiong Qin, and Tai-De Tan. The direct and indirect co2 rebound effect for private cars in china. Energy Policy, 100:149-161, 2017.

[37] Pengxiang Zhao, Dominik Bucher, Henry Martin, and Martin Raubal. A clustering-based framework for understanding individuals' travel mode choice behavior. In The Annual International Conference on Geographic Information Science, pages 7794. Springer, 2019. 